Original article

\title{
The association between body mass index and immunohistochemical subtypes in breast cancer
}

\author{
Suleyman Sahin a , Gokmen U. Erdem ${ }^{\text {d }}$, Fatih Karatas a, Aydin Aytekin ${ }^{\mathrm{a}}$, Ali R. Sever , \\ Yavuz Ozisik ${ }^{b}$, Kadri Altundag ${ }^{\text {b, * }}$ \\ a Department of Medical Oncology, Diskapi Training and Research Hospital, Ankara, Turkey \\ ${ }^{\mathrm{b}}$ Department of Medical Oncology, Hacettepe University Cancer Institute, Ankara, 06100, Turkey \\ ${ }^{c}$ Department of Radiology, Hacettepe University School of Medicine, Ankara, Turkey \\ d Department of Medical Oncology, Numune Training and Research Hospital, Ankara, Turkey
}

\section{A R T I C L E I N F O}

\section{Article history:}

Received 24 January 2016

Received in revised form

19 September 2016

Accepted 26 September 2016

Available online 15 October 2016

\section{Keywords:}

Breast cancer

Body mass index

Immunohistochemical subtypes

Relation

Premenopausal

Postmenopausal

\begin{abstract}
A B S T R A C T
Background: Body mass index (BMI) is defined as a poor prognostic factor in patients with breast cancer (BC). However, there are controversial results regarding the various effects of BMI on BC, hence the exact pathophysiology of the relation between obesity and $\mathrm{BC}$ is still under debate, and remains unclear. This paper aims to investigate the association between BMI at presentation and BC subtypes defined according to the immunohistochemical classification in both premenopausal and postmenopausal patients with BC. Patients and methods: This study is a retrospective and explorative analysis of the 3767 female BC patients from a single center. All patients' BMI at the time of initial diagnosis and tumor demographics were recorded. BMI was stratified into 3 groups as normal-weighted (BMI $<25 \mathrm{~kg} / \mathrm{m}^{2}$ ), over-weighted $\left(B M I=25-29.9 \mathrm{~kg} / \mathrm{m}^{2}\right)$, and obese $\left(B M I \geq 30 \mathrm{~kg} / \mathrm{m}^{2}\right)$. Immunohistochemical classification of the tumors was categorized into 4 groups as follows; luminal-like, HER2/luminal-like, HER2-like, and triplenegative according to the ER/PR and HER2 status. Distribution of Immunohistochemical subtypes, tumor characteristics, and overall survival (OS) analysis were evaluated according to the BMI groups in both premenopausal and postmenopausal patients.

Results: Median BMI of premenopausal and postmenopausal patients was $25.5\left(\mathrm{~kg} / \mathrm{m}^{2}\right)$ and $28.8\left(\mathrm{~kg} / \mathrm{m}^{2}\right)$, respectively $(\mathrm{P}<0.001)$. In parallel with the increasing age, patients were more obese at diagnosis in both premenopausal $(P<0.001)$ and postmenopausal period $(P<0.001)$. Triple-negative subtype was significantly more frequent in premenopausal patients with BMI $\geq 30 \mathrm{~kg} / \mathrm{m}^{2}$ compared to BMI $<30 \mathrm{~kg} / \mathrm{m}^{2}$ $(\mathrm{P}=0.007)$. Additionally, premenopausal patients with $\mathrm{BMI} \geq 30 \mathrm{~kg} / \mathrm{m}^{2}$ had less common luminal-like subtype $(P=0.033)$ and more frequently presented with higher tumor stage $(P=0.012)$ and tumor grade $(P=0.004)$ compared to patients with $B M I<25 \mathrm{~kg} / \mathrm{m}^{2}$. On the other hand, premenopausal patients with BMI $<25 \mathrm{~kg} / \mathrm{m}^{2}$ had significantly more ER-positive tumors $(\mathrm{P}<0.001)$ and lower stages of disease $(\mathrm{P}=0.01)$ compared to their counterparts with $\mathrm{BMI} \geq 25 \mathrm{~kg} / \mathrm{m}^{2}$. Premenopausal obese patients with triple-negative $(P=0.001)$ and luminal-like subtype $(P=0.002)$ had significantly shorter OS duration compared to overweight counterparts. HER2/luminal-like subtype was found to be significantly greater in postmenopausal overweight patients $(\mathrm{P}=0.005)$. However, BMI had no any other significant effect on survival and immunohistochemical subtypes in postmenopausal patients. Multivariate analysis revealed that triple-negative subtype, grade III tumor, BMI $\geq 30 \mathrm{~kg} / \mathrm{m}^{2}, \mathrm{~T} 3-4(\mathrm{P}<0.001)$, nodal involvement, metastatic disease, and lymphovascular involvement were significantly associated with poorer $\mathrm{OS}$.

Conclusion: Our data indicated that BMI was an independent factor in patients with BC, with an association indicating a decreased incidence for luminal-like subtype and increased incidence for triplenegative subtype among premenopausal patients. However, this significance was not found in postmenopausal patients. Accordingly, a plausible etiological heterogeneity in BC might play a role among immunohistochemical subtypes in every life stage of women.
\end{abstract}

(c) 2016 Elsevier Ltd. All rights reserved.

\footnotetext{
* Corresponding author. Fax: +90 3123242009.

E-mail address: altundag66@yahoo.com (K. Altundag).
} 


\section{Introduction}

Obesity has been a major health problem worldwide since its prevalence is increasing rapidly after 1980s [1] and it is a wellrecognized risk factor for metabolic syndrome, cardiovascular disease, diabetes mellitus and various cancers including breast cancer (BC) [2]. The role of Body Mass Index (BMI) and its effect on the prognosis in patients with $\mathrm{BC}$ has been evaluated with a great interest for many years. Despite controversial results of some studies, obesity has been regarded as a poor prognostic factor in BC [3]. A recent analysis of the Women's Health Initiative randomized study has revealed that women who were overweight and obese were associated with an increased risk of BC compared to those with normal weight [4]. Most of the previous epidemiological studies have shown a positive association between obesity and postmenopausal $\mathrm{BC}$ risk and an inverse relation between obesity and premenopausal BC risk [5]. This low risk in premenopausal period may be due to some endogenous hormonal factors and higher number of anovulatory menstrual cycles in obese premenopausal women [6]. By contrast, the high risk in postmenopausal period might be explained by increased concentrations of circulating estrogens since the adipose tissue is the main source of estrogens in postmenopausal period. Additionally, endogenous hormones such as estrogen and progesterone were reported to have mitogenic and morphogenic effects on mammalian epithelial cells by paracrine effect [7]. However, the main pathophysiology of this complex association between BMI and BC risk in women's life periods has not yet been clarified.

$\mathrm{BC}$ is a heterogeneous disease with different clinical presentations and classified into several histological subtypes according to the estrogen receptor (ER), progesterone receptor (PR) and human epidermal growth factor receptor 2 (HER2) status [8]. The effect of obesity on BC prognosis changes in compliance with the clinical and histopathological characteristics of disease, such as menopausal status and tumor subtypes [3].

Today, possible effects of obesity on immunohistochemical subtypes of BC (luminal-like, HER2/luminal-like, HER2-like, triplenegative) are still unclear in pre and postmenopausal patients [9]. Herein we aimed to determine the association between BMI and immunohistochemical subtypes of $\mathrm{BC}$ whether the obesity has any manipulating role in the incidence of developing different $\mathrm{BC}$ subtypes in pre and postmenopausal patients.

\section{Materials and methods}

\subsection{Data collection and enrollment}

In this retrospective and explorative analysis, we reached to medical records of 4413 Turkish female BC patients being followed between 1994 and 2015 in Hacettepe University Institute of Oncology. Of the 4413 patients, 646 were excluded due to unknown BMI data $(n=296)$, missing receptor status $(n=203)$ or ductal carcinoma in situ histology $(\mathrm{n}=147)$. Remaining 3767 patients were enrolled in this study (Fig. 1). Tumor demographics including, tumor stage, tumor grade, lymphovascular invasion (LVI), perineural invasion (PNI), ER, PR, and HER2 status, and nodal involvement were found from original histopathology reports. BMI and other clinical information of all patients were carefully recorded during the follow up period. Final status of the patients was found by using the hospital death records notification system.

\subsection{Definition of tumor subtypes and BMI}

HER2 status was determined by immunohistochemical (IHC) staining. Tumors having score of 3 were considered as
HER2-positive. Tumors scoring $2(+)$ for HER2 expression were subsequently analyzed by fluorescence in situ hybridization (FISH) test and were considered as HER2-positive if HER2 amplification was present in FISH test. ER and PR nuclear staining $\geq 1 \%$ were accepted as ER and/or PR-positive by IHC evaluation according to the ASCO/CAP - guidelines [10]. Immunohistochemical subtypes were categorized into 4 groups as luminal-like (ER and/or PRpositive and HER2-negative), HER2/luminal-like (ER and/or PRpositive and HER2-positive), HER2-like (ER and PR-negative and HER2-positive) and triple-negative (ER, PR and HER2-negative) according to the ER/PR and HER2 status [11]. BMI was calculated by the formula of weight $(\mathrm{kg}) /$ height ${ }^{2}\left(\mathrm{~m}^{2}\right)$ and then stratified into 3 groups as normal-weighted (BMI $<25 \mathrm{~kg} / \mathrm{m}^{2}$ ), over-weighted $\left(B M I=25-29.9 \mathrm{~kg} / \mathrm{m}^{2}\right)$ and obese $\left(B M I \geq 30 \mathrm{~kg} / \mathrm{m}^{2}\right)$, according to World Health Organization Classification - 2012. As the number of patients with BMI $<18.5 \mathrm{~kg} / \mathrm{m}^{2}(\mathrm{n}=29)$ was too small, we have not constituted a separate group for underweight patients and this group of patients were combined with the normal-weighted group.

Distribution of clinical features, immunohistochemical and histological subtypes, age, grade, LVI, PNI, nodal status, tumor stage, clinical stage and cumulative overall survival (OS) probability were analyzed according to BMI stratification.

\subsection{Statistical analysis}

All statistical analysis was performed by using the computer program of 'Statistical Package for The Social Sciences' version 18.0 for Windows (SPSS, Inc., Chicago, IL, USA). P-value equal or less than 0.05 was accepted as statistically significant in all analysis. For descriptive analysis, categorical variables were defined as frequency and distributions with percentages and quantitative variables were presented as median, minimum, and maximum values. Categorical variables were analyzed by using Chi-square or Fisher exact test. Kruskal-Wallis and a following Mann-Whitney U test were used if data were not normally distributed. The differences among the groups were evaluated by post hoc analysis. Survival analysis was performed according to the Kaplan-Meier Method. Log-rank statistics was used to compare the subgroup analysis. OS was defined as the period from the diagnosis until the date of death or the date of last visit. Factors identified by univariate analysis were subsequently evaluated in Cox-regression analysis for the purpose of determining the independent predictors of survival.

\section{Results}

\subsection{Patient characteristics}

Of the 3767 patients, 1834 were premenopausal (48.7\%), 1666 (\% 44.2) were postmenopausal and 267 (7.1\%) were perimenopausal. All women aged greater than 60 years, women who had bilateral ovariectomy operation and women aged younger than 60 years with an intact uterus not receiving hormone replacement therapy and being amenorrheic for at least one year before the $\mathrm{BC}$ diagnosis were defined as postmenopausal according to the National Comprehensive Cancer Network-guidelines version 1.2016 [12]. The number of normal-weighted, overweight and obese patients in premenopausal group was 954 (45.4\%), 710 (33.8\%) and 437 (20.8\%), respectively and in postmenopausal group this was 347 (20.8\%), 638 (38.3\%) and 681 (40.9\%), respectively. Median age of all patients was 48.6 years (range: 18.1-92.1). According to the menopausal status, median age of premenopausal and postmenopausal patients was 42.5 (range: $18.1-58.8$ ) and 58.1 years (range: 31.1-92.1), respectively. In parallel with the aging, patients were more obese at diagnosis in both premenopausal and postmenopausal period $(\mathrm{P}<0.001$ vs. $\mathrm{P}<0.001$, respectively). Median 


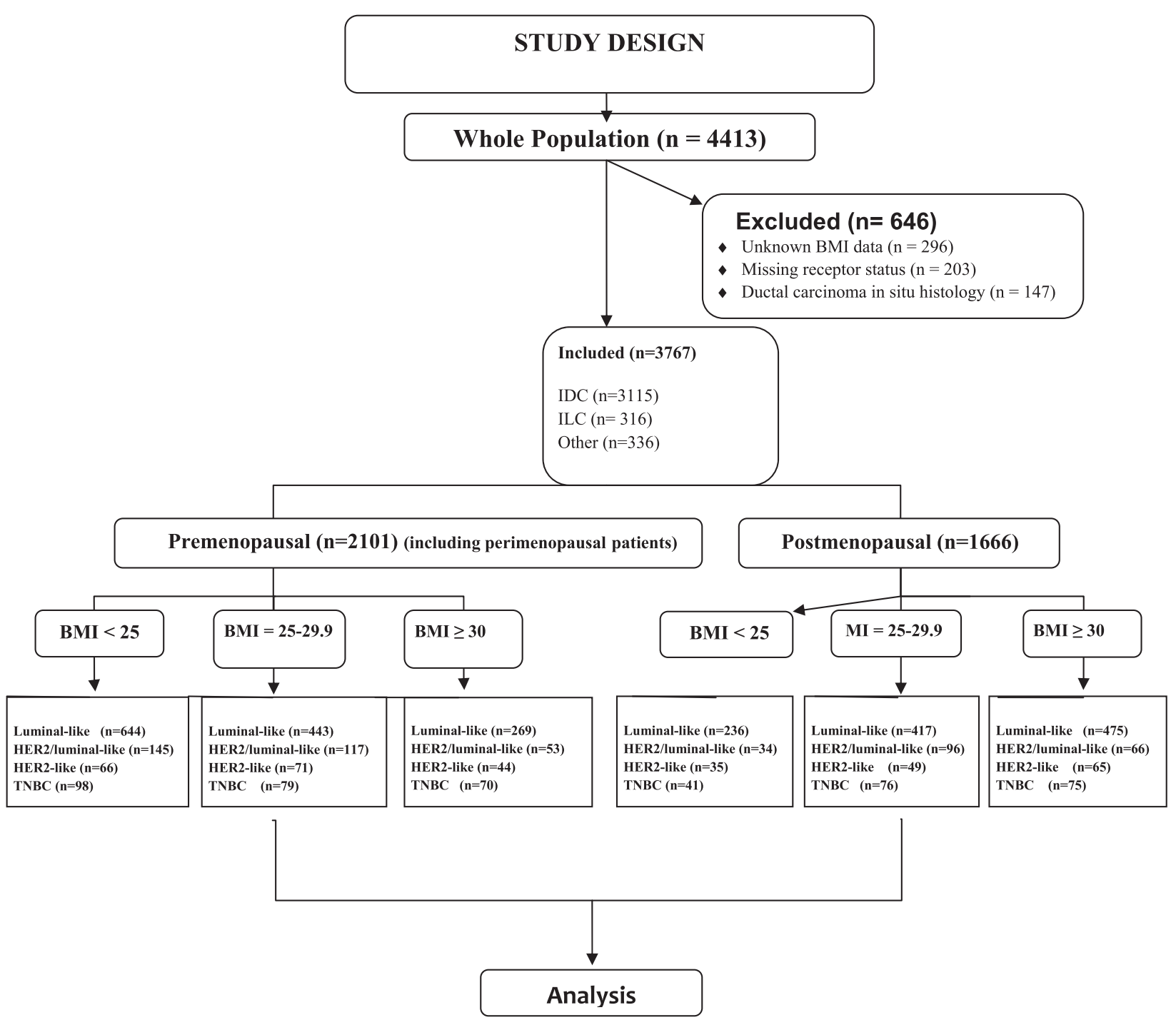

$\mathrm{BMI}=$ Body mass index, $\mathrm{IDC}=$ Invasive ductal carcinoma, $\mathrm{ILC}=$ Invasive lobular carcinoma, $\mathrm{TNBC}=$ Triple-negative breast cancer, HER2 = Human epidermal growth factor receptor-2

Fig. 1. Consort diagram.

BMI of postmenopausal patients was significantly greater than premenopausal patients $\left(28.8 \mathrm{~kg} / \mathrm{m}^{2}\right.$ vs. $\left.25.5 \mathrm{~kg} / \mathrm{m}^{2}, \mathrm{P}<0,001\right)$. Patient characteristics were shown in Table 1.

\subsection{Immunohistochemical subtypes according to BMI groups}

Distribution of immunohistochemical subtypes and histopathological features according to BMI groups were shown in each separate table for premenopausal and postmenopausal patients (Tables 2, 3). Triple-negative subtype was significantly more frequent in premenopausal patients with BMI $\geq 30 \mathrm{~kg} / \mathrm{m}^{2}$ (obese) compared to $\mathrm{BMI}<30 \mathrm{~kg} / \mathrm{m}^{2}(\mathrm{P}=0.007)$. In addition, obese premenopausal patients had less common luminal-like subtype $(P=0.033)$, and more frequently presented with higher tumor stage $(P=0.012)$ and tumor grade $(P=0.004)$ when compared to patients with $\mathrm{BMI}<25 \mathrm{~kg} / \mathrm{m}^{2}$. By contrast, premenopausal patients with $\mathrm{BMI}<25 \mathrm{~kg} / \mathrm{m}^{2}$ had significantly more ER-positive tumors $(\mathrm{P}<0.001)$ and lower stages of disease $(\mathrm{P}=0.01)$ compared to their counterparts with BMI $\geq 25 \mathrm{~kg} / \mathrm{m}^{2}$. In order to minimize the different or imprecise definitions of the subgroups that might lead to conflicting results of the relation between BMI and prognosis and biology of BC, we also used the methodology of Eichholzer et al. [5] to parallelize the our evaluation. For premenopausal group, patients with $\mathrm{BMI} \geq 30 \mathrm{~kg} / \mathrm{m}^{2}$ had significantly more ER negative/PR negative tumors compared to those with BMI $<25 \mathrm{~kg} \mathrm{~kg} / \mathrm{m}^{2}$ $(P=0.001)$. Similarly, there was no significant finding regarding the association between BMI and BC subtypes in postmenopausal patients.

Overweight premenopausal patients had more nod-positive disease $(P=0.022)$ and HER2-like subtype $(P=0.041)$ compared to normal-weighted patients. In postmenopausal patients, HER2/ luminal-like subtype was found to be significantly associated with overweight BMI $(P=0.005)$. However, BMI had no other significant effect on immunohistochemical subtypes in postmenopausal patients. Besides, no other relationship was observed between BMI and LVI, PNI, histological subtype, ER, PR, and HER2 status in both pre and postmenopausal patients.

of note, for luminal-like group, we performed an additional classification according to tumor grade, with the goal of defining luminal A-like group (grade 1-2), and luminal B/HER2-negative- 
Table 1

Patient characteristics.

\begin{tabular}{|c|c|}
\hline Median age (year) & $48.6(18.1-92.1)$ \\
\hline Pre & $42.5(18.1-58.8)$ \\
\hline Post & $58.1(31.1-92.1)$ \\
\hline Median BMI (kg/m²) & $27.0(15.5-55.5)\left({ }^{*} \mathrm{P}<0.001\right)$ \\
\hline Pre & $25.5(15.6-51.0)$ \\
\hline Post & $28.8(15.5-55.5)$ \\
\hline \multicolumn{2}{|l|}{ Menopausal status (n) } \\
\hline Pre & $1834(48.7 \%)$ \\
\hline Post & $1666(44.2 \%)$ \\
\hline Peri & $267(7.1)$ \\
\hline \multicolumn{2}{|l|}{ Histological subtype } \\
\hline IDC & 3115 (82.7\%) \\
\hline ILC & $316(8.4 \%)$ \\
\hline Other & $336(8.9 \%)$ \\
\hline \multicolumn{2}{|l|}{ LVI } \\
\hline Positive & $1094(29.0 \%)$ \\
\hline Negative & $2673(71.0 \%)$ \\
\hline \multicolumn{2}{|l|}{ PNI } \\
\hline Positive & $416(11.0 \%)$ \\
\hline Negative & $3351(89.0 \%)$ \\
\hline \multicolumn{2}{|l|}{ Grade } \\
\hline 1 & 397 (11.7\%) \\
\hline 2 & $1547(45.6 \%)$ \\
\hline 3 & $1451(42.7 \%)$ \\
\hline \multicolumn{2}{|c|}{ Immunohistochemical subtype } \\
\hline Luminal-like & $2484(65.9 \%)$ \\
\hline Grade $1 / 2$ & $1563(62.9 \%)$ \\
\hline Grade 3 & $655(26.4 \%)$ \\
\hline Unknown & $266(10.7 \%)$ \\
\hline HER2/Luminal-like & $514(13.6 \%)$ \\
\hline HER2-like & $330(8.8 \%)$ \\
\hline Triple negative & $439(11.7 \%)$ \\
\hline \multicolumn{2}{|l|}{$\mathbf{T}$} \\
\hline 1 & $1233(33.6 \%)$ \\
\hline 2 & $1757(47.9 \%)$ \\
\hline 3 & $481(13.1 \%)$ \\
\hline 4 & $196(5.4 \%)$ \\
\hline \multicolumn{2}{|r|}{ (20) } \\
\hline 0 & $1618(44.3 \%)$ \\
\hline 1 & $1058(29.0 \%)$ \\
\hline 2 & $543(14.9 \%)$ \\
\hline 3 & $435(11.9 \%)$ \\
\hline \multicolumn{2}{|l|}{ Clinical stage } \\
\hline I & $823(21.8 \%)$ \\
\hline II & $1571(41.7 \%)$ \\
\hline III & $969(25.7 \%)$ \\
\hline IV & $335(8.9 \%)$ \\
\hline Missing & $69(1.8)$ \\
\hline \multicolumn{2}{|c|}{ Adjuvant chemotherapy } \\
\hline Pre & $1777(84.8 \%)$ \\
\hline Post & $1050(63.7 \%)$ \\
\hline \multicolumn{2}{|l|}{ Adjuvant radiotherapy } \\
\hline Pre & $1477(76.5 \%)$ \\
\hline Post & $1034(68.8 \%)$ \\
\hline \multicolumn{2}{|c|}{ Adjuvant endocrine therapy } \\
\hline Pre & $1511(78.3 \%)$ \\
\hline Post & $1202(80.0 \%)$ \\
\hline \multicolumn{2}{|l|}{ Surgery } \\
\hline MRM & $1250(33.2 \%)$ \\
\hline BCS & $2292(60.8 \%)$ \\
\hline Not operated & $225(6.0 \%)$ \\
\hline
\end{tabular}

${ }^{*} \mathrm{P}$ value is from Mann Whitney U test.

BMI = Body mass index, Pre $=$ Premenopausal, Post $=$ postmenopausal, IDC = Invasive ductal carcinoma, ILC = Invasive lobular carcinoma, HER2 = Human epidermal growth factor receptor-2, LVI = Lymphovascular invasion, $\mathrm{PNI}=$ Perineural invasion, $\mathrm{T}=$ Tumor stage, $\mathrm{N}=$ Nodal stage, $\mathrm{MRM}=$ Modified radical mastectomy, $\mathrm{BCS}=$ Breast conserving surgery.

like group (grade 3 ), since luminal-like group is a very heterogeneous group and important to distinguish as they include subtyperelated outcome. After reclassifying the luminal-like group as luminal A-like (grade 1-2), and luminal B/HER2-negative-like (grade 3), we observed that the low rate of luminal-like subtype in obese premenopausal patients was associated with luminal A-like group ( $P=0.005)$, and there was no significant difference in the rate of luminal B/HER2-negative-like group in obese premenopausal patients. The classification of luminal-like group by grading system and its distribution according to BMI was shown in Table 4.

\subsection{Treatment characteristics}

Of the 3767 patients, 3137 were treated in adjuvant setting, 295 in neoadjuvant setting, and 335 in metastatic setting. A total of 2292 patients underwent surgery of modified radical mastectomy, while 1250 patients were treated with breast conserving surgery. Among BMI groups, no significant difference in relation to chemotherapy and radiotherapy treatment was found between pre and postmenopausal patients. However, premenopausal obese patients appeared to receive less hormonal therapy compared to their counterparts with $\mathrm{BMI}<30 \mathrm{~kg} / \mathrm{m}^{2}(\mathrm{P}<0.001)$.

\subsection{Survival outcomes}

Median follow-up time was 48.6 months for premenopausal group and 47.7 months for postmenopausal group. In whole population, patients with $\mathrm{BMI} \geq 30 \mathrm{~kg} / \mathrm{m}^{2}$ had significantly shorter median OS duration as compared to patients with BMI $<30 \mathrm{~kg} / \mathrm{m}^{2}$ $(\mathrm{P}=0.002)$ (Fig. 2). However, this significance with regard to BMI strata was only observed in premenopausal period $(\mathrm{P}<0.001)$, while not observed in postmenopausal period $(P=0.95)$ (Figs. 3,4$)$. Median OS durations according to the immunohistochemical subtypes among premenopausal patients were significantly shorter for luminal-like (182 months, 95\% CI, 142.7-222.6, $\mathrm{P}=0.002$ ) and triple-negative subtype (111 months, 95\% CI, 78.9-143.2, $\mathrm{P}=0.001$ ) in patients with BMI $\geq 30 \mathrm{~kg} / \mathrm{m}^{2}$ compared to normal and overweight patients (Figs. 5, 6). When reclassifying the luminal-like group according to tumor grade (grade $1-2$, or grade 3 ), as luminal A-like and luminal B/HER2-negative-like, the survival durations of obese premenopausal patients in luminal A-like versus luminal B/HER2-negative-like group were 182 versus 97 months, respectively $(\mathrm{P}=0.001)$, indicating that the shorter OS in obese premenopausal patients was associated with luminal B/HER2negative-like group. By contrast, any significant relation between BMI and immunohistochemical subtypes in terms of OS was not observed in postmenopausal patients.

Prognostic factors associated with OS were evaluated in Univariate analysis. BMI $\geq 30 \mathrm{~kg} / \mathrm{m}^{2}(\mathrm{P}=0.002)$, triple-negative subtype ( $\mathrm{P}=0.002)$, HER2-positive tumor $(\mathrm{P}<0.008)$, ER-negative tumor $(\mathrm{P}<0.001)$, PR-negative tumor $(\mathrm{P}<0.001)$, hormone negativity $(\mathrm{P}<0.001)$, LVI $(\mathrm{P}<0.001)$, PNI $(\mathrm{P}=0.012)$, stage III-IV disease $(\mathrm{P}<0.001)$, T3-4 $(\mathrm{P}<0.001)$, nodal involvement $(\mathrm{P}<0.001)$, M1 disease $(\mathrm{P}<0.001)$, and grade III tumor $(\mathrm{P}<0.001)$ were the factors affecting OS. Cox regression analysis revealed that triple-negative subtype, grade III tumor, BMI $\geq 30 \mathrm{~kg} / \mathrm{m}^{2}, \mathrm{~T} 3-4$, nodal involvement, M1 disease, and LVI were the independent predictors of OS (Table 5).

\section{Discussion}

Despite previous numerous studies, there is not yet a common consensus regarding the relationship between $\mathrm{BMI}$ and $\mathrm{BC}$, as the outcomes of the studies searching the effect of BMI on BC are conflicting. In this present study, obesity was found to be associated with an increased incidence of triple-negative subtype and decreased incidence of luminal-like subtype among premenopausal patients, with a significantly shorter overall survival. After reclassifying the luminal-like group according to tumor grade (grade 1-2, or grade 3), as luminal A-like and luminal B/HER2negative-like, the shorter OS in obese premenopausal patients 
Table 2

Distribution of histopathological features by BMI groups, for premenopausal patients.

\begin{tabular}{|c|c|c|c|c|c|}
\hline & \multicolumn{5}{|l|}{ BMI $\left(\mathrm{kg} / \mathrm{m}^{2}\right)$} \\
\hline & $<25(\%)$ & $25-29.9(\%)$ & $\geq 30(\%)$ & Total (\%) & *P \\
\hline Age (year) & $40.4(18.1-55.8)$ & $43.2(19.6-57.3)$ & $45.3(24.6-58.8)$ & $42.5(18.1-58.8)$ & $<0.001+$ \\
\hline \multicolumn{6}{|l|}{ Histological subtype } \\
\hline IDC & $810(84.9)$ & $603(85.0)$ & $370(84.7)$ & $1783(84.8)$ & \multirow[t]{3}{*}{0.943} \\
\hline ILC & $77(8.0)$ & $55(7.7)$ & $34(7.7)$ & $166(7.9)$ & \\
\hline Other & $67(7.1)$ & $52(7.3)$ & $33(7.6)$ & $152(7.3)$ & \\
\hline \multicolumn{6}{|l|}{ LVI } \\
\hline Positive & $297(31.1)$ & $212(29.9)$ & $141(32.3)$ & $650(30.9)$ & \multirow[t]{2}{*}{0.683} \\
\hline Negative & $657(68.9)$ & $498(70.1)$ & $296(67.7)$ & $1451(69.1)$ & \\
\hline \multicolumn{6}{|l|}{ PNI } \\
\hline Positive & $92(9.6)$ & $86(12.1)$ & $42(9.6)$ & $220(10.5)$ & \multirow[t]{2}{*}{0.214} \\
\hline Negative & $862(90.4)$ & $624(87.9)$ & $395(90.4)$ & $1881(89.5)$ & \\
\hline \multicolumn{6}{|l|}{ Grade } \\
\hline I-II & 499 (58.7) & 347 (52.7) & $195(49.4)$ & $1041(54.7)$ & \multirow[t]{2}{*}{0.004} \\
\hline III & $351(41.3)$ & $311(47.3)$ & $200(50.6)$ & $862(45.3)$ & \\
\hline \multicolumn{6}{|l|}{ Luminal-like } \\
\hline Yes & $644(67.5)$ & $443(62.4)$ & 269 (61.6) & $1356(64.5)$ & \multirow[t]{2}{*}{0.033} \\
\hline No & $310(32.5)$ & $267(37.6)$ & $168(38.4)$ & $745(35.5)$ & \\
\hline \multicolumn{6}{|l|}{ HER2/luminal-like } \\
\hline Yes & $145(15.2)$ & $117(16.5)$ & $53(12.1)$ & $315(15.0)$ & \multirow[t]{2}{*}{0.130} \\
\hline No & $809(84.8)$ & $593(83.5)$ & $384(87.9)$ & $1786(85.0)$ & \\
\hline \multicolumn{6}{|l|}{ HER2-like } \\
\hline Yes & $66(6.9)$ & $71(10.0)$ & $44(10.1)$ & $181(8.6)$ & \multirow[t]{2}{*}{0.041} \\
\hline No & $888(93.1)$ & $639(90.0)$ & 393 (89.9) & $1920(91.4)$ & \\
\hline \multicolumn{6}{|l|}{ Triple-negative } \\
\hline Yes & $98(10.3)$ & $79(11.1)$ & $70(16.0)$ & $247(11.8)$ & 0.007 \\
\hline No & $856(89.7)$ & $631(88.9)$ & $367(84.0)$ & $1854(88.2)$ & \\
\hline $\mathbf{T}$ & & & & & \\
\hline $1-2$ & $766(82.0)$ & $553(79.2)$ & $312(75.0)$ & $1631(79.6)$ & 0.012 \\
\hline $3-4$ & $168(18.0)$ & $145(20.8)$ & $104(25.0)$ & $417(20.4)$ & \\
\hline Nodal involvement & & & & & \\
\hline Positive & $512(54.7)$ & $429(61.5)$ & $237(56.6)$ & $1178(57.4)$ & 0.022 \\
\hline Negative & $424(45.3)$ & $269(38.5)$ & $182(43.4)$ & $875(42.6)$ & \\
\hline Clinical stage & & & & & \\
\hline $\mathrm{I}-\mathrm{II}$ & $630(67.3)$ & $434(61.5)$ & $254(60.0)$ & $1318(63.8)$ & 0.01 \\
\hline III-IV & $306(32.7)$ & $272(38.5)$ & $169(40.0)$ & $747(36.2)$ & \\
\hline ER status & & & & & \\
\hline Positive & $757(79.4)$ & $520(73.2)$ & $303(69.3)$ & $1580(75.2)$ & $<0.001$ \\
\hline Negative & $197(20.6)$ & $190(26.8)$ & $134(30.7)$ & $521(24.8)$ & \\
\hline PR status & & & & & \\
\hline Positive & $720(75.5)$ & $518(73.0)$ & $303(69.3)$ & $1541(73.3)$ & 0.054 \\
\hline Negative & $234(24.5)$ & $192(27.0)$ & $134(30.7)$ & $560(26.7)$ & \\
\hline HER2 receptor status & & & & & \\
\hline Positive & $212(22.2)$ & $188(26.5)$ & $98(22.4)$ & $498(23.7)$ & 0.101 \\
\hline Negative & $742(77.8)$ & $525(73.5)$ & $339(77.6)$ & $1603(76.3)$ & \\
\hline Hormone receptor statu & & & & & \\
\hline ER positive/PR positive & $687(72.0)$ & $478(67.3)$ & $283(64.8)$ & $1448(68.9)$ & 0.013 \\
\hline ER negative/PR negative & $164(17.2)$ & $150(21.1)$ & $114(26.1)$ & $428(20.4)$ & 0.001 \\
\hline Triple-negativity & & & & & \\
\hline Yes & $98(10.3)$ & $79(11.1)$ & $70(16.0)$ & $247(11.8)$ & 0.007 \\
\hline No & $856(89.7)$ & $631(88.9)$ & $367(84.0)$ & $1854(88.2)$ & \\
\hline Chemotherapy & & & & & \\
\hline Yes & $796(83.8)$ & $606(85.6)$ & $375(85.8)$ & $1777(84.8)$ & 0.48 \\
\hline No & $154(16.2)$ & $102(14.4)$ & $62(14.2)$ & $318(15.2)$ & \\
\hline Hormonal therapy & & & & & \\
\hline Yes & $771(81.3)$ & 549 (77.9) & 309 (71.5) & $1629(78.1)$ & $<0.001$ \\
\hline No & $177(18.7)$ & $156(22.1)$ & $123(28.5)$ & 456 (21.9) & \\
\hline Radiotherapy & & & & & \\
\hline Yes & $718(75.5)$ & $553(79.0)$ & $328(76.5)$ & 1599 (76.9) & 0.24 \\
\hline No & $233(24.5)$ & $147(21.0)$ & $101(23.5)$ & $481(23.1)$ & \\
\hline
\end{tabular}

${ }^{*} \mathrm{P}$ values are from the Chi-square test unless otherwise indicated. + Kruskal-Wallis test.

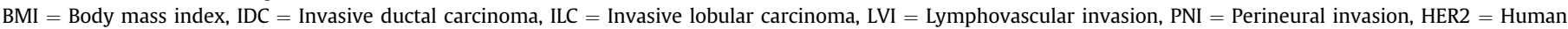
epidermal growth factor receptor-2, $\mathrm{T}=$ Tumor stage, $\mathrm{ER}=$ Estrogen receptor, $\mathrm{PR}=$ Progesterone receptor.

was found to be related to luminal B/HER2-negative-like group (182 vs. 97 months, $\mathrm{P}=0.001$ ).

Obesity has been accepted as an important risk factor for postmenopausal BC [8]. However, in a chemoprevention study it was reported that $B M I$ had a significant relation with increased risk of $\mathrm{BC}$ among premenopausal women $>35$ years of ages, but not for postmenopausal women [13]. Furthermore, a meta-analysis designed by Cheraghi et al. showed that BMI had no impact on $\mathrm{BC}$ incidence during premenopausal period and also indicated that the role of obesity on $\mathrm{BC}$ is not clinically important despite the significant statistics [14].

Our study results demonstrated that obese premenopausal patients, but not postmenopausal group, had a higher tumor grade, larger tumor stage, and more advanced clinical stage. Likewise, 
Table 3

Distribution of histopathological features by BMI groups, for postmenopausal patients.

\begin{tabular}{|c|c|c|c|c|c|}
\hline & \multicolumn{5}{|l|}{ BMI $\left(\mathrm{kg} / \mathrm{m}^{2}\right)$} \\
\hline & $<25(\%)$ & $25-29.9(\%)$ & $\geq 30(\%)$ & Total (\%) & *P-value \\
\hline Age (year) & $56.7(31.4-83.7)$ & $58.2(31.1-92.1)$ & $58.9(33.2-89.4)$ & $58.1(31.1-92.1)$ & $<0.001+$ \\
\hline \multicolumn{6}{|l|}{ Histological subtype (n) } \\
\hline IDC & $278(80.1)$ & $500(78.3)$ & $554(81.3)$ & 1332 (79.9) & \multirow[t]{3}{*}{0.435} \\
\hline ILC & $35(10.0)$ & $57(8.9)$ & $58(8.5)$ & $150(9.0)$ & \\
\hline Other & $34(9.8)$ & $81(12.7)$ & $69(10.1)$ & $184(11.0)$ & \\
\hline \multicolumn{6}{|l|}{ LVI } \\
\hline Positive & $86(24.8)$ & $170(26.6)$ & $188(27.6)$ & $444(26.7)$ & \multirow[t]{2}{*}{0.626} \\
\hline Negative & $261(75.2)$ & $468(73.4)$ & $493(72.4)$ & $1222(73.3)$ & \\
\hline \multicolumn{6}{|l|}{ PNI } \\
\hline Positive & $44(12.7)$ & 76 (11.9) & $76(11.2)$ & $196(11.8)$ & \multirow[t]{2}{*}{0.766} \\
\hline Negative & $303(87.3)$ & $562(88.1)$ & $605(88.8)$ & $1470(88.2)$ & \\
\hline \multicolumn{6}{|l|}{ Grade } \\
\hline I-II & $189(61.4)$ & $356(62.6)$ & $358(58.2)$ & $903(60.5)$ & \multirow[t]{2}{*}{0.292} \\
\hline III & $119(38.6)$ & $213(37.4)$ & $257(41.8)$ & $589(39.5)$ & \\
\hline \multicolumn{6}{|l|}{ Luminal-like } \\
\hline Yes & $236(68.0)$ & $417(65.4)$ & $475(69.8)$ & $1128(67.7)$ & \multirow[t]{2}{*}{0.232} \\
\hline No & $111(32.0)$ & $221(34.6)$ & $206(30.2)$ & $538(32.3)$ & \\
\hline \multicolumn{6}{|l|}{ HER2/luminal-like } \\
\hline Yes & $34(9.8)$ & $96(15.0)$ & $66(9.7)$ & $196(11.8)$ & \multirow[t]{2}{*}{0.005} \\
\hline No & $313(90.2)$ & $542(85.0)$ & $615(90.3)$ & $1470(88.2)$ & \\
\hline \multicolumn{6}{|l|}{ HER2-like } \\
\hline Yes & $35(10.1)$ & $49(7.7)$ & $65(9.5)$ & $149(8.9)$ & \multirow[t]{2}{*}{0.348} \\
\hline No & $312(89.9)$ & $589(92.3)$ & $616(90.5)$ & $1517(91.1)$ & \\
\hline \multicolumn{6}{|l|}{ Triple-negative } \\
\hline Yes & $41(11.8)$ & 76 (11.9) & $75(11.0)$ & $192(11.5)$ & 0.862 \\
\hline No & $306(88.2)$ & $562(88.1)$ & $606(89.0)$ & $1474(88.5)$ & \\
\hline $\mathbf{T}$ & & & & & \\
\hline $1-2$ & $278(83.5)$ & $522(84.7)$ & $558(83.4)$ & $1358(83.9)$ & 0.785 \\
\hline $3-4$ & $55(16.5)$ & $94(15.3)$ & $111(16.6)$ & $260(16.1)$ & \\
\hline Nodal involvement & & & & & \\
\hline Positive & $172(51.8)$ & $317(51.5)$ & $369(56.4)$ & $858(53.6)$ & 0.168 \\
\hline Negative & $160(48.2)$ & $298(48.5)$ & $285(43.6)$ & $743(46.4)$ & \\
\hline Clinical stage & & & & & \\
\hline I-II & $235(69.1)$ & $400(64.3)$ & $441(65.7)$ & 1076 (65.9) & 0.320 \\
\hline III-IV & 105 (30.9) & $222(35.7)$ & $230(34.3)$ & $557(34.1)$ & \\
\hline ER status & & & & & \\
\hline Positive & $255(73.5)$ & $492(77.1)$ & $524(76.9)$ & $1271(76.3)$ & 0.385 \\
\hline Negative & $92(26.5)$ & 146 (22.9) & $157(23.1)$ & $395(23.7)$ & \\
\hline PR status & & & & & \\
\hline Positive & $226(65.1)$ & $430(67.4)$ & $476(69.9)$ & $1132(67.9)$ & 0.281 \\
\hline Negative & $121(34.9)$ & $208(32.6)$ & $205(30.1)$ & $534(32.1)$ & \\
\hline HER2 receptor status & & & & & \\
\hline Positive & $70(20.2)$ & $145(22.7)$ & $132(19.4)$ & $347(20.8)$ & 0.309 \\
\hline Negative & $277(79.8)$ & $493(77.3)$ & $549(80.6)$ & $1319(79.2)$ & \\
\hline Hormone receptor status & & & & & \\
\hline ER positive/PR positive & $210(60.5)$ & $409(64.1)$ & $459(67.4)$ & $1078(64.7)$ & 0.085 \\
\hline ER negative/PR negative & $76(21.9)$ & $125(19.6)$ & $140(20.6)$ & $341(20.1)$ & 0.690 \\
\hline Triple-negative & & & & & \\
\hline Yes & $41(11.8)$ & $76(11.9)$ & $75(11.0)$ & $192(11.5)$ & 0.862 \\
\hline No & $306(88.2)$ & $562(88.1)$ & $606(89.0)$ & $1474(88.5)$ & \\
\hline Chemotherapy & & & & & 0.77 \\
\hline Yes & $223(65.2)$ & 397 (62.9) & $430(63.6)$ & $1050(63.7)$ & \\
\hline No & $119(34.8)$ & $234(37.1)$ & $246(36.4)$ & $599(36.3)$ & \\
\hline Hormonal therapy & & & & & \\
\hline Yes & $269(78.2)$ & $508(80.5)$ & $538(79.7)$ & 1315 (79.7) & 0.69 \\
\hline No & $75(21.8)$ & $123(19.5)$ & $137(20.3)$ & $335(20.3)$ & \\
\hline Radiotherapy & & & & & \\
\hline Yes & $220(64.3)$ & $434(69.1)$ & 477 (71.7) & $1131(69.2)$ & 0.055 \\
\hline No & $122(35.7)$ & $194(30.9)$ & $188(28.3)$ & $504(30.8)$ & \\
\hline
\end{tabular}

${ }^{*} \mathrm{P}$ values are from the Chi-square test unless otherwise indicated. + Kruskal-Wallis test.

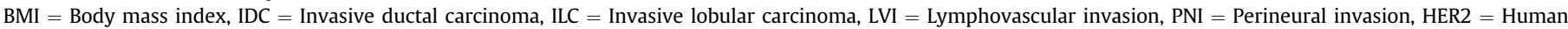
epidermal growth factor receptor-2, $\mathrm{T}=$ Tumor stage, $\mathrm{ER}=$ Estrogen receptor, $\mathrm{PR}=$ Progesterone receptor.

Eichholzer et al. reported that BMI had a positive relation with TNM stage, grading and tumor stage [5]. Biglia et al. also reported that overweight and obese women had significantly greater tumor stage at diagnosis compared to normal-weighted women [9]. Similar trials investigating the association between BMI, tumor grade and tumor size have also reported a significant correlation between BMI and poor survival $[15,16]$. The relation between BMI and molecular subtypes in $\mathrm{BC}$ has been investigated in a few previous populationbased studies, indicating conflicting results. For instance, Caroline $\mathrm{BC}$ study demonstrated no association between BMI and the risk of basal-like BC [17], whereas the Polish BC study found an increased risk in basal-like BC among premenopausal women [18]. Yang et al. reported that obesity in younger women was related to the risk of developing ER-positive or PR-positive tumors, but not to the risk of 
Table 4

The classification of luminal-like group by grade, and its distribution according to BMI.

\begin{tabular}{|c|c|c|c|c|c|}
\hline & \multicolumn{5}{|l|}{ BMI $\left(\mathrm{kg} / \mathrm{m}^{2}\right)$} \\
\hline & $<25(\%)$ & $25-29.9(\%)$ & $\geq 30(\%)$ & Total (\%) & ${ }^{*} \mathrm{P}$ \\
\hline \multicolumn{6}{|l|}{ Luminal-like premenopausal patients } \\
\hline Yes & $644(67.5)$ & $443(62.4)$ & $269(61.6)$ & $1356(64.5)$ & 0.033 \\
\hline No & $310(32.5)$ & $267(37.6)$ & $168(38.4)$ & $745(35.5)$ & \\
\hline Grade 1-2 (luminal A-like) & & & & & 0.005 \\
\hline Yes & $410(46.2)$ & $266(39.7)$ & $153(37.9)$ & $829(42.3)$ & \\
\hline No & $477(53.8)$ & $404(60.3)$ & $251(62.1)$ & $1132(57.7)$ & \\
\hline Grade 3 (luminal B/HER2-negative-like) & & & & & 0.656 \\
\hline Yes & $167(18.8)$ & $137(20.4)$ & $83(20.5)$ & 387 (19.7) & \\
\hline No & $720(81.2)$ & $533(79.6)$ & $321(79.5)$ & $1574(80.3)$ & \\
\hline \multicolumn{6}{|l|}{ Luminal-like postmenopausal patients } \\
\hline Yes & $34(9.8)$ & $417(65.4)$ & $475(69.8)$ & $1128(67.7)$ & 0.232 \\
\hline No & $313(90.2)$ & $221(34.6)$ & $206(30.2)$ & $538(32.3)$ & \\
\hline \multicolumn{6}{|l|}{ Grade 1-2 } \\
\hline Yes & 157 (49.5) & $280(47.2)$ & $297(47.1)$ & 734 (47.7) & 0.757 \\
\hline No & $160(50.5)$ & $313(52.8)$ & 333 (52.9) & $806(52.3)$ & \\
\hline \multicolumn{6}{|l|}{ Grade 3} \\
\hline Yes & 49 (15.5) & $92(15.5)$ & $127(20.2)$ & $268(17.4)$ & 0.06 \\
\hline No & $268(84.5)$ & $501(84.5)$ & $503(79.8)$ & $1272(82.6)$ & \\
\hline
\end{tabular}

${ }^{*}$ P values are from the Chi-square test unless otherwise indicated.

$\mathrm{BMI}=$ Body mass index, HER2 = Human epidermal growth factor receptor-2.

triple-negative subtype, suggesting that BMI seems to be more associated with hormone receptor positive tumors rather than triple negative breast cancer (TNBC) [19]. In our study, premenopausal obesity was significantly correlated with triple-negative subtype, similar to the findings of previous study by Turkoz et al. who showed that premenopausal obese patients had an increased rate of hormone-negative BC [20]. Similarly, Petekkaya et al. indicated a significant increase in ER/PR negative tumors among premenopausal BC patients with BMI $\geq 25 \mathrm{~kg} / \mathrm{m}^{2}$ [21]. Likewise, Daling et al. [22], Milikan et al. [17] and Mahle et al. [23] found a positive association between BMI and hormone receptor-negative tumors. While Gaudet et al. [24] reported a positive relation between BMI and risk of developing TNBC in patients under 56 years of age,

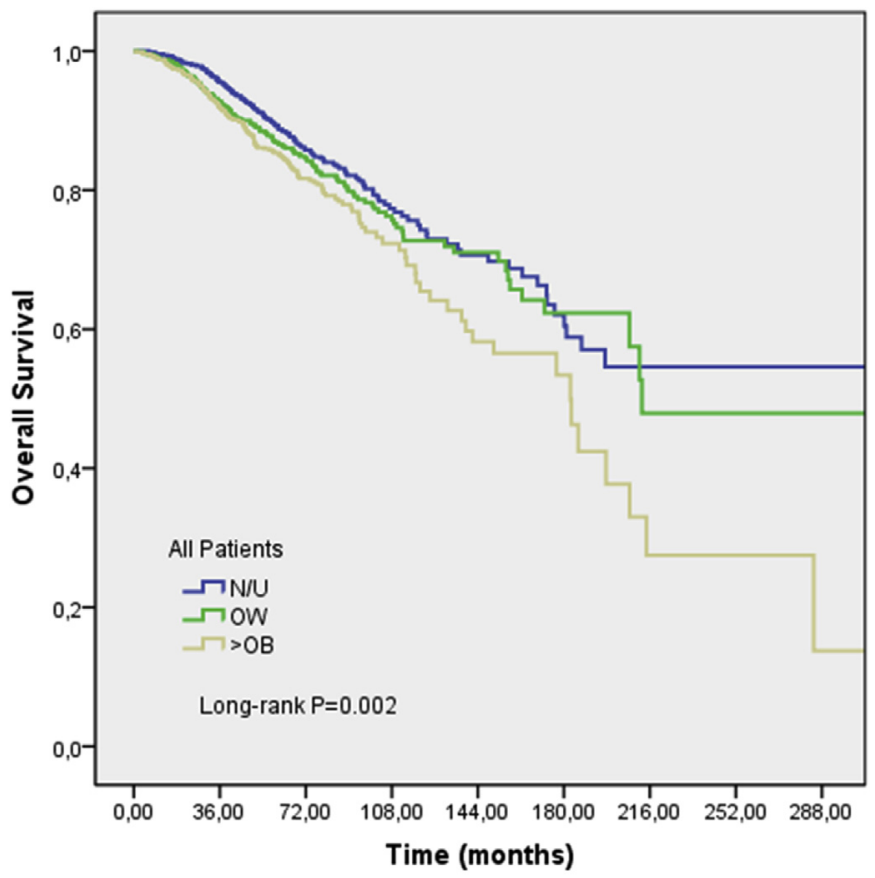

$\mathrm{N} / \mathrm{U}=$ Normal or underweight, $\mathrm{OW}=$ Overweight, $\mathrm{OB}=$ Obese

Fig. 2. Overall survival by BMI Groups, for all patients.
Biglia et al. found no statistically significant association between BC subtypes (luminal A, B, HER2-like and basal-like) and BMI in pre and postmenopausal women [9]. Sueta et al. demonstrated that BMI and its change was found to be related to increased risk of luminal-like and TNBC among postmenopausal Japanese women [25].

While several studies reported a positive and compatible relationship between body size and hormone-positive BC [26-28], some other studies analyzing the relation between BMI and TNBC showed incompatible outcomes. Among them, Phipps et al. reported an increased risk for both TNBC (odds ratio (OR) $=1.35 ; 95 \%$ confidence interval (CI): 0.92-1.99) and ER-positive tumor $(\mathrm{OR}=1.39$; $95 \% \mathrm{CI}: 1.22-1.58)$ in patients with higher BMI values

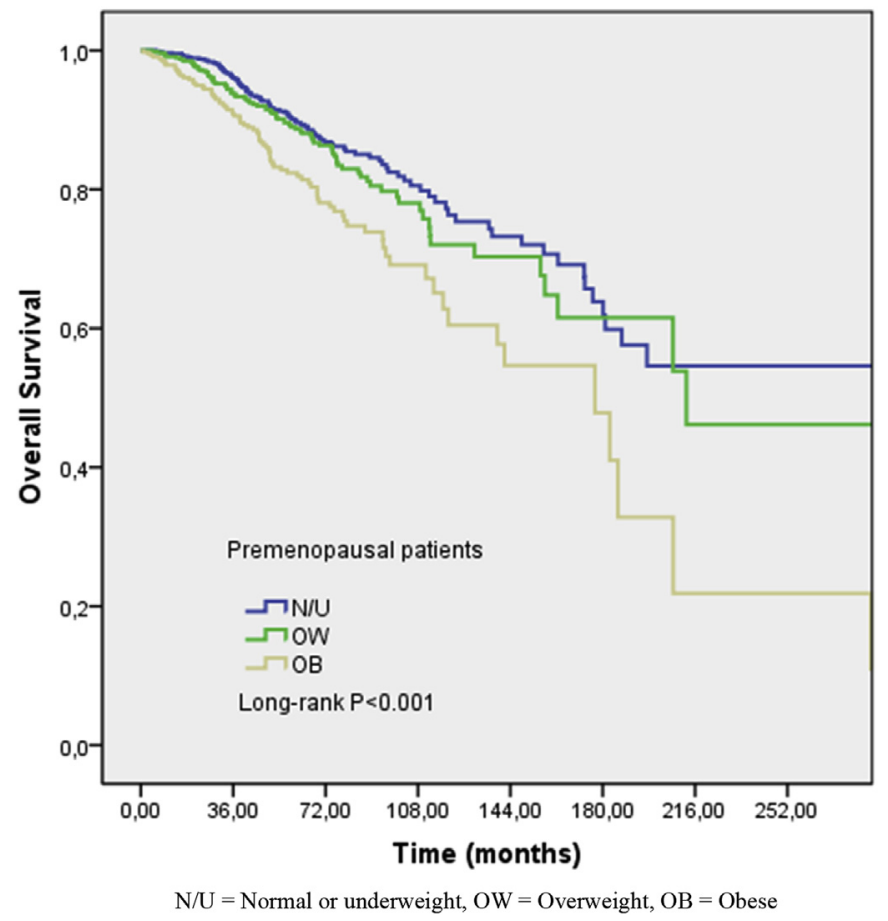

Fig. 3. Overall survival by BMI groups, for premenopausal patients. 


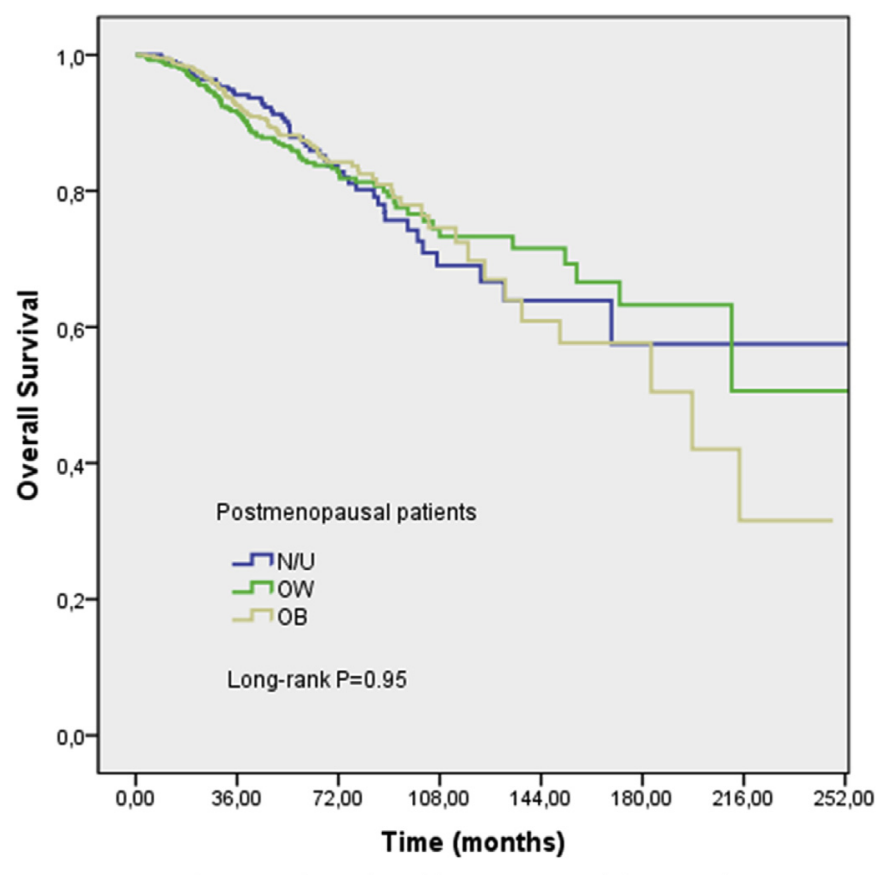

$\mathrm{N} / \mathrm{U}=$ Normal or underweight, $\mathrm{OW}=$ Overweight, $\mathrm{OB}=$ Obese

Fig. 4. Overall survival by BMI groups for postmenopausal patients.

[29]. The fact that our study showed an increased incidence for triple-negative subtype and a decreased incidence for luminal-like subtype among premenopausal obese patients were quite consistent. As a result, these controversial outcomes in literature may suggest that immunohistochemical and molecular subtypes are not only associated with BMI in both pre and postmenopausal patients

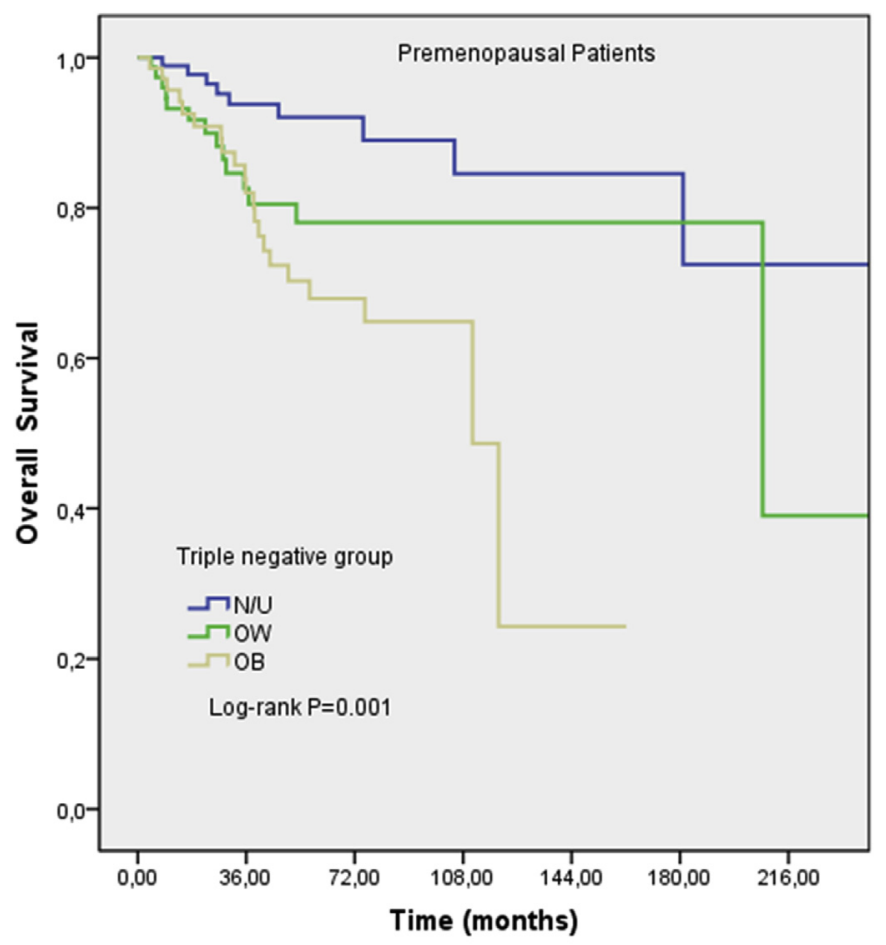

$\mathrm{N} / \mathrm{U}=$ Normal or underweight, $\mathrm{OW}=$ Overweight, $\mathrm{OB}=$ Obese

Fig. 5. Survival for premenopausal patients with Triple-negative subtype.

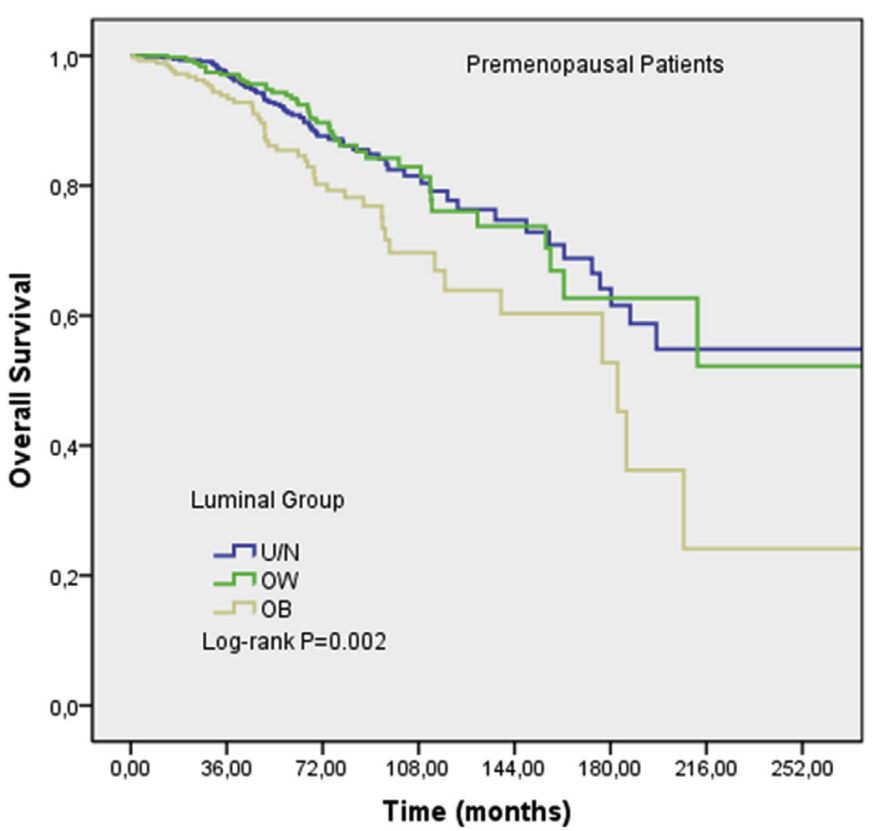

$\mathrm{N} / \mathrm{U}=$ Normal or underweight, $\mathrm{OW}=$ Overweight, $\mathrm{OB}=$ Obese

Fig. 6. Survival for premenopausal patients with Luminal-like subtype.

but also indicate a distinct etiology among immunohistochemical and molecular subtypes in every life stage of women.

Overall, our study demonstrated that BMI was inversely and significantly associated with OS in luminal-like and triple-negative subtype among premenopausal patients, reflecting the similar result of previous studies [22,30,31]. Besides, the detrimental effect of increased BMI on survival in our study was consistent with the recent pooled analysis of eight prospective neoadjuvant breast cancer trials by Fontanella $C$ et al. who have reported significantly decreased mean DFS and OS for obese patients with luminal-like and TNBC [32]. Besides, we showed that BMI $\geq 30 \mathrm{~kg} / \mathrm{m}^{2}$ was associated with 1.5-fold increased risk in mortality among patients with $\mathrm{BC}$ at the time of diagnosis, similar to the findings of a cohort study by Chen X et al. who reported that BC patients with BMI $\geq 30 \mathrm{~kg} / \mathrm{m}^{2}$ had a hazard rate of 1.55 (95\% CI 1.10-2.17) for total mortality in comparison to patients with normal BMI [15]. Likewise, several cohort studies have reported that obesity is associated with poorer overall survival for patients with BC in every stage of life [22,33-35].

Aside from its retrospective nature, our study had some limitations. Despite the fact that BMI is capable to show the obesity index, it is not appropriate in the use of determining the ratio of muscle and fat tissue in the body. However, BMI is mostly used as a predicting parameter of body fat in human. Besides, central obesity which is the measurement of waist - hip ratio has been suggested as a risk factor for premenopausal BC, regardless of BMI [36]. However, we did not have the measurements of waist and hip circumferences in order to identify central obesity in patients with normal BMI. Moreover, we did not have $\mathrm{Ki}-67$ test results that might have affected the optimal immunohistochemical and molecular classification of BC subtypes. By contrast, the fact that our database and medical records were carefully collected between 1994 and 2015 from 3767 eligible patients with known tumor features and BMI information was the most powerful side of our study. Moreover, self-reported BMI information was not included in our database in order to obtain the most accurate BMI data. In addition to this, the numbers of triple-negative and HER2-like subtype were relatively greater in our population than previous studies investigating the association between BMI and BC [5]. 
Table 5

Variables associated with overall survival in Univariate and Multivariate analysis.

\begin{tabular}{|c|c|c|c|c|}
\hline \multirow[t]{2}{*}{ Variables } & \multirow{2}{*}{$\frac{\text { Univariate Analysis }}{\mathrm{P}}$} & \multicolumn{3}{|c|}{ Cox-regression analysis } \\
\hline & & $\mathrm{P}$ & HR & $95 \% \mathrm{CI}$ \\
\hline ER-negative tumor & $<0.001$ & & & \\
\hline PR-negative tumor & $<0.001$ & & & \\
\hline Hormone-negative & $<0.001$ & & & \\
\hline HER2-positive tumor & $<0.008$ & & & \\
\hline Triple-negative subtype & 0.002 & 0.003 & 1.658 & $1.6581 .188-2.314$ \\
\hline Premenopausal & 0.238 & & & \\
\hline $\mathrm{BMI} \geq 30 \mathrm{~kg} / \mathrm{m}^{2}$ & 0.002 & 0.009 & 1.505 & $1.101-2.059$ \\
\hline $\mathrm{PNI}$ & 0.012 & & & \\
\hline LVI & $<0.001$ & 0.049 & 1.303 & $1.002-1.695$ \\
\hline Stage III-IV disease & $<0.001$ & & & \\
\hline T3-4 & $<0.001$ & $<0.001$ & 1.862 & $1.428-2.427$ \\
\hline $\mathrm{N}$-positive & $<0.001$ & $<0.001$ & 1.959 & $1.428-2.689$ \\
\hline M1 & $<0.001$ & $<0.001$ & 6.925 & $5.180-9.260$ \\
\hline Grade III tumor & $<0.001$ & 0.005 & 1.452 & $1.121-1.880$ \\
\hline
\end{tabular}

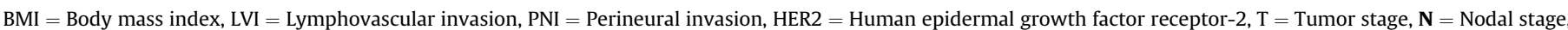
$\mathrm{ER}=$ Estrogen receptor, $\mathrm{PR}=$ Progesterone receptor, $\mathrm{CI}=$ Confidence interval, $\mathrm{HR}=$ Hazard ratio, $\mathrm{M}=$ Metastasis.

In conclusion, obesity was found to be related to an increased risk of ER/PR negative tumors and correlated with poor median overall survival among premenopausal patients with breast cancer. However, despite the fact that obesity has been previously accepted as a poor prognostic risk factor for overall survival among postmenopausal patients, it was highly important to note that we showed no significant association between body mass index and breast cancer in postmenopausal patients. Additionally, as obesity has been reported to be an adverse prognostic factor of survival in patients with breast cancer, our findings indicated that higher BMI $\left(\geq 30 \mathrm{~kg} / \mathrm{m}^{2}\right)$ was found the independent predictor of BC mortality. Finally, specific risk for each immunohistochemical subtype may not also be associated with BMI in both pre and postmenopausal patients. Therefore, these conflicting results suggest that alongside the BMI, there might be more additional factors affecting BC and immunohistochemical subtypes.

\section{Conflicts of interest}

The authors indicated no potential conflict of interest.

\section{References}

[1] Alka Garg NG, Kumar Lalit. Study of body mass index and premenopausal or postmenopausal breast cancer. Int J Sci Study 2015;3(1):116-9.

[2] Rudat V, Birido N, Tuwaijri S, Al-Abbadi MA. Body mass index and breast cancer risk: a retrospective multi-institutional analysis in Saudi Arabia. Adv Breast Cancer Res 2013;02(01):7-10.

[3] Kogawa T, Fouad TM, Wei C, Masuda H, Kai K, Fujii T, et al. Association of body mass index changes during neoadjuvant chemotherapy with pathologic complete response and clinical outcomes in patients with locally advanced breast cancer. J Cancer 2015;6(4):310-8.

[4] Neuhouser ML, Aragaki AK, Prentice RL, Manson JE, Chlebowski R, Carty CL et al. Overweight, obesity, and postmenopausal invasive breast cancer risk: a secondary analysis of the women's health initiative randomized clinical trials. JAMA Oncol 2015;1(5):611-21.

[5] Eichholzer M, Huang DJ, Modlasiak A, Schmid SM, Schotzau A, Rohrmann S, et al. Impact of body mass index on prognostically relevant breast cancer tumor characteristics. Breast Care 2013;8(3):192-8.

[6] Nichols HB, Trentham-Dietz A, Egan KM, Titus-Ernstoff L, Holmes MD, Bersch AJ, et al. Body mass index before and after breast cancer diagnosis: associations with all-cause, breast cancer, and cardiovascular disease mortality. Cancer Epidemiol Biomark Prev Publ Am Assoc Cancer Res Cosponsored by Am Soc Prev Oncol 2009;18(5):1403-9.

[7] Suzuki R, Saji S, Toi M. Impact of body mass index on breast cancer in accordance with the life-stage of women. Front Oncol 2012;2:123.

[8] Xing Peng, Li JG, Jin Feng, Zhao Ting-Ting, Liu Qun, Dong Hui-Ting, et al. Prognostic significance of body mass index in breast cancer patients with hormone receptor-positive tumours after curative surgery. Clin Invest Med 2013;36(6):297-305.
[9] Biglia N, Peano E, Sgandurra P, Moggio G, Pecchio S, Maggiorotto F, et al. Body mass index (BMI) and breast cancer: impact on tumor histopathologic features, cancer subtypes and recurrence rate in pre and postmenopausal women. Gynecol Endocrinol Off J Int Soc Gynecol Endocrinol 2013;29(3):263-7.

[10] Hammond ME, Hayes DF, Dowsett M, Allred DC, Hagerty KL, Badve S, et al. American Society of Clinical Oncology/College of American Pathologists guideline recommendations for immunohistochemical testing of estrogen and progesterone receptors in breast cancer. J Clin Oncol Off J Am Soc Clin Oncol 2010:28(16):2784-95.

[11] Onitilo AA, Engel JM, Greenlee RT, Mukesh BN. Breast cancer subtypes based on ER/PR and Her2 expression: comparison of clinicopathologic features and survival. Clin Med Res 2009;7(1-2):4-13.

[12] National Comprehensive Cancer Network. Bone Cancer (Version 1.2016). http://www.nccn.org/professionals/physician_gls/pdf/breast.pdf. Accessed March 2016

[13] Cecchini RS, Costantino JP, Cauley JA, Cronin WM, Wickerham DL, Land SR, et al. Body mass index and the risk for developing invasive breast cancer among high-risk women in NSABP P-1 and STAR breast cancer prevention trials. Cancer Prev Res 2012;5(4):583-92.

[14] Cheraghi Z, Poorolajal J, Hashem T, Esmailnasab N, Doosti Irani A. Effect of body mass index on breast cancer during premenopausal and postmenopausal periods: a meta-analysis. PloS One 2012;7(12):e51446.

[15] Chen X, Lu W, Zheng W, Gu K, Chen Z, Zheng Y, et al. Obesity and weight change in relation to breast cancer survival. Breast Cancer Res Treat 2010;122(3):823-33.

[16] Rosenberg L, Czene K, Hall P. Obesity and poor breast cancer prognosis: an illusion because of hormone replacement therapy? Br J cancer 2009;100(9): 1486-91.

[17] Millikan RC, Newman B, Tse CK, Moorman PG, Conway K, Dressler LG, et al. Epidemiology of basal-like breast cancer. Breast Cancer Res Treat 2008;109(1):123-39.

[18] Yang XR, Sherman ME, Rimm DL, Lissowska J, Brinton LA, Peplonska B, et al. Differences in risk factors for breast cancer molecular subtypes in a population-based study. Cancer Epidemiol Biomark Prev Publ Am Assoc Cancer Res Cosponsored by Am Soc Prev Oncol 2007;16(3):439-43.

[19] Yang XR, Chang-Claude J, Goode EL, Couch FJ, Nevanlinna H, Milne RL, et al. Associations of breast cancer risk factors with tumor subtypes: a pooled analysis from the Breast Cancer Association Consortium studies. J Natl Cancer Inst 2011;103(3):250-63.

[20] Turkoz FP, Solak M, Petekkaya I, Keskin O, Kertmen N, Sarici F, et al. The prognostic impact of obesity on molecular subtypes of breast cancer in premenopausal women. J BUON 2013;18(2):335-41.

[21] Petekkaya I, Sahin U, Gezgen G, Solak M, Yuce D, Dizdar O, et al. Association of breast cancer subtypes and body mass index. Tumori 2013:99(2):129-33.

[22] Daling JR, Malone KE, Doody DR, Johnson LG, Gralow JR, Porter PL. Relation of body mass index to tumor markers and survival among young women with invasive ductal breast carcinoma. Cancer 2001;92(4):720-9.

[23] Maehle BO, Tretli S, Skjaerven R, Thorsen T. Premorbid body weight and its relations to primary tumour diameter in breast cancer patients; its dependence on estrogen and progesteron receptor status. Breast Cancer Res Treat 2001;68(2):159-69.

[24] Gaudet MM, Press MF, Haile RW, Lynch CF, Glaser SL, Schildkraut J, et al. Risk factors by molecular subtypes of breast cancer across a population-based study of women 56 years or younger. Breast Cancer Res Treat 2011;130(2): 587-97.

[25] Sueta Aiko, Ito Hidemi, Islam Tania, Hosono Satoyo, Watanabe Miki, Hirose Kaoru, et al. Differential impact of body mass index and its change on 
the risk of breast cancer by molecular subtype: a case-control study in Japanese women. SpringerPlus 2012;(39):1.

[26] Enger SM, Ross RK, Paganini-Hill A, Carpenter CL, Bernstein L. Body size, physical activity, and breast cancer hormone receptor status: results from two case-control studies. Cancer Epidemiol Biomark Prev Publ Am Assoc Cancer Res Cosponsored by Am Soc Prev Oncol 2000;9(7):681-7.

[27] Colditz GA, Rosner BA, Chen WY, Holmes MD, Hankinson SE. Risk factors for breast cancer according to estrogen and progesterone receptor status. J Natl Cancer Inst 2004:96(3):218-28.

[28] Suzuki R, Orsini N, Saji S, Key TJ, Wolk A. Body weight and incidence of breast cancer defined by estrogen and progesterone receptor status-a meta-analysis. Int J Cancer J Int du cancer 2009;124(3):698-712.

[29] Phipps AI, Chlebowski RT, Prentice R, McTiernan A, Stefanick ML, WactawskiWende J, et al. Body size, physical activity, and risk of triple-negative and estrogen receptor-positive breast cancer. Cancer Epidemiol Biomark Prev Publ Am Assoc Cancer Res Cosponsored by Am Soc Prev Oncol 2011:20(3):454-63.

[30] Whiteman MK, Hillis SD, Curtis KM, McDonald JA, Wingo PA, Marchbanks PA. Body mass and mortality after breast cancer diagnosis. Cancer Epidemiol Biomarkers Prev Publ Am Assoc Cancer Res Cosponsored by Am Soc Prev Oncol 2005;14(8):2009-14.
[31] Calle EE, Rodriguez C, Walker-Thurmond K, Thun MJ. Overweight, obesity, and mortality from cancer in a prospectively studied cohort of U.S. adults. N. Engl Med 2003;348(17):1625-38.

[32] Fontanella C, Lederer B, Gade S, Vanoppen M, Blohmer JU, Costa SD, et al Impact of body mass index on neoadjuvant treatment outcome: a pooled analysis of eight prospective neoadjuvant breast cancer trials. Breast Cancer Res Treat 2015;150(1):127-39.

[33] Kroenke CH, Chen WY, Rosner B, Holmes MD. Weight, weight gain, and survival after breast cancer diagnosis. J Clin Oncol Off J Am Soc Clin Onco 2005;23(7):1370-8.

[34] Berclaz G, Li S, Price KN, Coates AS, Castiglione-Gertsch M, Rudenstam CM et al. Body mass index as a prognostic feature in operable breast cancer: the International Breast Cancer Study Group experience. Ann Oncol Off J Eur Soc Med Oncol/ESMO 2004;15(6):875-84.

[35] Ewertz M, Jensen MB, Gunnarsdottir KA, Hojris I, Jakobsen EH, Nielsen D, et al Effect of obesity on prognosis after early-stage breast cancer. J Clin Oncol Off J Am Soc Clin Oncol 2011;29(1):25-31.

[36] Harvie M, Hooper L, Howell AH. Central obesity and breast cancer risk: a systematic review. Obes Rev Off J Int Assoc Study Obes 2003;4(3): 157-73. 